\title{
Depth Wise Variability of Available N, P and K at Different Locations under Rice - Pea- Sugarcane Cropping System in Meerut District (U.P)
}

Vipin Kumar, Satendra Kumar, Akansha Singh, B.P. Dhyani, U.P. Shahi, Mukesh Kumar, Purushottam, R.I. Navsare

10.18805/IJARe.A-5908

\begin{abstract}
Background: The research is based on the fertility status of the soil being affected year by year so here is a strategy to save the soil fertility level or soil health by the use of a leguminous cropping system.

Methods: A study was conducted during 2018-2021 in the Meerut district of western Uttar Pradesh to evaluate the soil characterization of the rice - pea- sugarcane cropping system. The soil samples were analyzed for various parameters in the laboratory. The status of available NPK in soils and other soil properties like pH, electrical conductivity (EC) and organic carbon (OC) content were assessed. Result: Results reveal that the soils of the study area were neutral to strongly alkaline in reaction and non-saline in nature. Nutrient status regarding to the available nutrient in the surface $(0-15 \mathrm{~cm})$ and subsurface $(15-30 \mathrm{~cm})$ depth of soil indicate that soils are low in available $\mathrm{N}$ and medium in available $\mathrm{P}$ and $\mathrm{K}$ in surface and subsurface. A positive and significant correlation of NPK with organic matter content was found while significant and negative correlations exist between the soil $\mathrm{pH}$.
\end{abstract}

Key words: Fertilizer, Pea, Rice, Soil.

\section{INTRODUCTION}

Cropping systems are designed and managed to grasp human goals so that they are purposeful systems. It includes every spatial and sequential aspect of managing an agricultural system. Cropping system-level study isn't only useful to know the overall sustainability of the agricultural system, but also it helps in generating many important parameters which are useful in global climate change impact assessment. Within the past, cropping systems were designed to maximize yield, but modern agriculture is progressively more concerned with promoting environmental sustainability in cropping systems.

The major cropping systems in western IGP (IndoGangetic Plane) are rice-wheat, sorghum-wheat, cottonwheat, pearl millet-rapeseed and mustard, maize-wheat, sugarcane-wheat, pigeon pea-wheat, groundnut-wheat, ricepea, rice-mustard/potato-black gram/mung bean. However, rice-wheat is that the predominant cropping system, occupying about 10 million ha. Soil fertility management becomes more complex in intensive cropping thanks to the residual effect of nutrients applied to the previous crops, a possible effect of legumes within the system, complementary and competitive interaction from the component crops and influence of crop residues left within the soil. The fashionable or chemical agriculture, which contains higher cropping intensity involving improved varieties, heavier inputs of fertilizer and water, increasing yields and accelerated removal of plant nutrients has added newer dimensions to fertility management. Nutrient removal under different cropping systems could even be variable since it depends on rooting pattern, the extent of biomass production and
Department of Soil Science and Agricultural Chemistry, Sardar Vallabhbhai Patel University of Agriculture and Technology, Meerut250 110, Uttar Pradesh, India.

Corresponding Author: Vipin Kumar, Department of Soil Science and Agricultural Chemistry, Sardar Vallabhbhai Patel University of Agriculture and Technology, Meerut-250 110, Uttar Pradesh, India. Email: vipinkumar291994@gmail.com

How to cite this article: Kumar, V., Kumar, S., Singh, A., Dhyani, B.P., Shahi, U.P., Kumar, M., Purushottam and Navsare, R.I. (2022). Depth Wise Variability of Available N, P and K at Different Locations under Rice - Pea- Sugarcane Cropping System in Meerut District (U.P). Indian Journal of Agricultural Research. DOI: 10.18805/IJARe.A-5908.

Submitted: 06-09-2021 Accepted: 14-12-2021 Online: 11-02-2022

superior absorption of the nutrient. Supported future fertility experiments conclusions that various parts of India, application of $\mathrm{N}$ at recommended dose is an advocate to every of the crop in a cropping system. Phosphorus management in cropping systems needs careful adjustment of $P$ fertilizer dose taking into account reasonably fertilizer, soil characteristics and their yield level, the extent of $P$ removal and growing environment. In a cropping system involving wheat, fertilizer $P$ dose to Kharif crop could even be reduced if preceding wheat has received $P$ in adequate amounts. Removal of $\mathrm{K}$ in proportion to $\mathrm{N}$ is incredibly high in cropping systems particularly those involving cereal and fodder crops. It's important to use $\mathrm{K}$ fertilizer at the recommended dose to care for soil fertility. The nonstop practice of cereal-cereal rotations within the Indo-Gangetic 
Depth Wise Variability of Available N, P and K at Different Locations under Rice - Pea- Sugarcane Cropping System in Meerut...

Plains (IGP) of India has caused a nutrient imbalance, soil degradation and intensive pest pressure (Ladha et al. 2003; Chauhan et al. 2012). This successively deteriorates crop productivity within the region, a trend that's increasingly evident within the IGP. With growing access to irrigation facilities, farmers within the IGP region have shown a higher preference input-responsive cereals than other crops (particularly pulses); thereby aggravating the issues related to low crop diversification (Ali and Gupta 2012). In comparison to rice-wheat and rice-fallow cropping systems, soils in rice-legume crop cycles with high input and diversity of organic materials have been found to contain higher concentrations of microbial biomass and enzymes. In the current situation, an intense cropping system that is not only highly productive and profitable but also stable over time and maintains soil fertility is crucial. The inclusion of pulses, oilseeds and vegetables is more helpful than cereal following cereal in the system. Furthermore, legumes are said to have a positive impact on soil fertility and aid in enhancing the production of subsequent rice crops.

\section{MATERIALS AND METHODS}

The study area falls in the Meerut district of western Uttar Pradesh. The area is situated at a latitude of $29^{\circ} 40^{\prime}$ North and longitude of $77^{\circ} 42^{\prime}$ East with an elevation of $237 \mathrm{~m}$ above mean sea level. Soil samples from two depths (0-15 and $15-30 \mathrm{~cm}$ ) from 48 locations with the use of GPS of Meerut district under different cropping patterns were collected with the help of an auger and stored in polythene bags. Collected soil samples were air-dried in shade crushed gently with a wooden roller and then pass through a $2.0 \mathrm{~mm}$ sieve to obtain a uniform representative sample. Samples were properly labeled with the aluminum tag and stored in polythene bags for analysis. The processed soil samples were analyzed by standard methods for $\mathrm{pH}$ and electrical conductivity (1:2 soil water suspensions), organic matter (Walkley and Black, 1934), available nitrogen (Subbiah and Asija, 1956), available phosphorus, available potassium (Jackson, 1973). All the analytical work was carried out in the laboratory of the Department of Soil Science, SVPUA and Tech, Meerut (U.P), India.

\section{RESULTS AND DISCUSSION}

The availability of N,P and K after harvesting of rice- pea cropping system the analysis was done which show some changing in soil properties. Data regarding the soil $\mathrm{pH}$ of surface and subsurface are presented in (Table 2). The soil samples of surface and subsurface were usually found normal to alkaline reaction and it varied from 7.21 to 8.10 and 7.25 to 8.12 , respectively (Table 2 ). A similar finding was observed by Rajeswar et al., (2009). The minimum pH of 7.21 at the surface was observed in the Alamgirpur village of the Parikshitgarh block, while the maximum $\mathrm{pH}$ of 8.10 was observed in Lawar Khas village of Daurala block. Similarly, in the subsurface, minimum $\mathrm{pH} 7.25$ and maximum $\mathrm{pH} 8.12$ were observed at both the same locations. Data presented in Table 2 shows that about $2.08 \%$ of the soil samples were found to be neutral, $54.17 \%$ were slightly alkaline and $43.75 \%$ was moderately alkaline at surface soil while at subsurface $2.08 \%$ of the soil samples were found to be saline, $41.66 \%$ were slightly alkaline and $56.25 \%$ were moderately alkaline. (Neutral: 6.6-7.3, Slightly alkaline: 7.47.8, Moderately alkaline: 7.9-8.4) This may be due to the influence of parent material, rainfall and topography (Thangaswamy et al., 2005). In other soil properties, electrical conductivity was also observed that the electrical conductivity of surface and subsurface soil varied from 0.17 to $0.56 \mathrm{dSm}^{-1}$ and 0.26 to $0.48 \mathrm{dSm}^{-1}$, respectively. Based on the limits suggested by Muhar et al., (1963) for judging the salt problem of soils, most of the samples were found normal (EC).

The organic carbon content in soil at the surface $(0-15$ $\mathrm{cm}$ ) ranged from 0.30 to 0.51 per cent with a mean value of 0.38 per cent, while at subsurface $(15-30 \mathrm{~cm})$, ranged from 0.19 to 0.42 per cent with a mean value of 0.28 per cent (Table 2) The highest organic carbon ( 0.51 per cent) was found in Sultannagar location and minimum ( 0.30 per cent) in Daurala location for surface soil $(0-15 \mathrm{~cm})$, while in subsurface soil $(15-30 \mathrm{~cm})$, maximum organic carbon $(0.42$ per cent) in Sultannagar location and minimum ( 0.19 per cent) in Daurala location was recorded. Based on the rating suggested by Singh et al. (2005), Most of the soil samples were low in organic carbon status at the surface, while subsurface soil was also low in organic carbon.

The available nitrogen content in surface $(0-15 \mathrm{~cm})$ and subsurface $(15-30 \mathrm{~cm})$ soils varied from 169.98 to 255.90 $\mathrm{kg} \mathrm{ha}^{-1}$ and 107.89 to $180.13 \mathrm{~kg} \mathrm{ha}^{-1}$, respectively (Table 3). The maximum available nitrogen 255.90 and $180.13 \mathrm{~kg} \mathrm{ha}^{-1}$ at surface and subsurface was found in Sultannagar village of Sururpur block and minimum 169.98 and $107.89 \mathrm{~kg} \mathrm{ha}^{-1}$ village Lawar Khas. Based on the rating suggested by Subbiah and Asija (1956) $97 \%$ of samples were low (<250 $\left.\mathrm{N} \mathrm{kg} \mathrm{ha}^{-1}\right)$ and $3 \%$ of samples were medium (250-500 N kg $\left.\mathrm{ha}^{-1}\right)$. Available nitrogen content was found to be maximum in surface horizons and decrease regularly with soil depth,

Table 1: Correlation studies between $\mathrm{OM}$ and $\mathrm{pH}$ with available $\mathrm{N}$, $\mathrm{P}$ and $\mathrm{K}$ under different locations.

\begin{tabular}{lcccc}
\hline & \multicolumn{2}{c}{$0-15 \mathrm{~cm}$ depth } & \multicolumn{2}{c}{$15-30 \mathrm{~cm}$ depth } \\
\cline { 2 - 5 } & $\mathrm{pH}$ & O.C & $\mathrm{pH}$ & O.C \\
\hline $\mathrm{pH}$ & 1.00 & & 1.00 & \\
O.C & $-0.211^{\mathrm{NS}}$ & 1.00 & $-0.230^{\mathrm{NS}}$ & 1.00 \\
Avail. N & $-0.243^{\mathrm{NS}}$ & $0.952^{* *}$ & $-0.236^{\mathrm{NS}}$ & $0.983^{* *}$ \\
Avail. P & $-0.176^{\mathrm{NS}}$ & $0.658^{* *}$ & $-0.185^{\mathrm{NS}}$ & $0.973^{* *}$ \\
Avail. K & $-0.238^{\mathrm{NS}}$ & $0.960^{* *}$ & $-0.303^{\mathrm{NS}}$ & $0.795^{* *}$ \\
\hline
\end{tabular}

*Significant $(p=0.05)$.

OC significantly and positively correlated with the above discussed soil properties $(r=0.952,0.658,0.960$ and subsurface, $r=0.983$, 0.973 and 0.795 respectively).

The analysis indicates that $\mathrm{pH}$ was negatively correlated with Available N, P and $K$ in surface $(0-15 \mathrm{~cm})$ and subsurface $(15-30 \mathrm{~cm})$. 
Depth Wise Variability of Available N, P and K at Different Locations under Rice - Pea- Sugarcane Cropping System in Meerut...

Table 2: Depth wise pH, O.C (\%) and E.C $\left(\mathrm{dS} \mathrm{m}^{-1}\right)$ of different location soil under rice -pea -sugarcane cropping system.

\begin{tabular}{|c|c|c|c|c|c|c|c|c|c|c|}
\hline \multicolumn{2}{|c|}{ Location } & \multicolumn{9}{|c|}{ Cropping system (Rice - pea - sugarcane) and Depth $(\mathrm{cm})$} \\
\hline \multirow{2}{*}{ Blocks name } & \multirow{2}{*}{ Village name } & \multicolumn{3}{|c|}{$\mathrm{pH}$} & \multicolumn{3}{|c|}{ O.C $(\%)$} & \multicolumn{3}{|c|}{ E.C dS m ${ }^{-1}$} \\
\hline & & $0-15$ & $15-30$ & Mean & $0-15$ & $15-30$ & Mean & $0-15$ & $15-30$ & Mean \\
\hline \multirow[t]{4}{*}{ Daurala } & Lawar Khas & 8.10 & 8.12 & 8.11 & 0.31 & 0.19 & 0.25 & 0.17 & 0.29 & 0.23 \\
\hline & Lawar Khas & 7.48 & 7.48 & 7.48 & 0.30 & 0.19 & 0.25 & 0.35 & 0.31 & 0.33 \\
\hline & Akhtyarpur & 7.79 & 7.81 & 7.80 & 0.30 & 0.20 & 0.25 & 0.42 & 0.48 & 0.45 \\
\hline & Chindauri & 7.80 & 7.80 & 7.80 & 0.44 & 0.35 & 0.40 & 0.32 & 0.42 & 0.37 \\
\hline \multirow[t]{4}{*}{ Shardhana } & Chabariya & 8.03 & 8.03 & 8.03 & 0.32 & 0.22 & 0.27 & 0.37 & 0.34 & 0.36 \\
\hline & Begumabad & 7.47 & 7.48 & 7.47 & 0.34 & 0.24 & 0.29 & 0.31 & 0.33 & 0.32 \\
\hline & Milak & 7.57 & 7.58 & 7.57 & 0.32 & 0.22 & 0.27 & 0.29 & 0.26 & 0.28 \\
\hline & Teherkiya & 8.08 & 8.10 & 8.09 & 0.42 & 0.32 & 0.37 & 0.37 & 0.35 & 0.36 \\
\hline \multirow[t]{4}{*}{ Sururpur } & Saroorpur Khurd & 7.87 & 7.89 & 7.88 & 0.36 & 0.26 & 0.31 & 0.36 & 0.41 & 0.39 \\
\hline & Kheri Kalan, & 7.70 & 7.71 & 7.70 & 0.32 & 0.22 & 0.27 & 0.31 & 0.36 & 0.34 \\
\hline & Harar & 7.49 & 7.49 & 7.49 & 0.38 & 0.27 & 0.33 & 0.32 & 0.29 & 0.31 \\
\hline & Sultannagar & 7.48 & 7.50 & 7.49 & 0.51 & 0.42 & 0.47 & 0.32 & 0.40 & 0.36 \\
\hline \multirow[t]{4}{*}{ Rohta } & Rasulpur & 7.94 & 7.95 & 7.94 & 0.41 & 0.31 & 0.36 & 0.30 & 0.33 & 0.32 \\
\hline & Rasna & 7.46 & 7.46 & 7.46 & 0.48 & 0.38 & 0.43 & 0.38 & 0.29 & 0.34 \\
\hline & Bhadaura & 7.74 & 7.75 & 7.74 & 0.39 & 0.29 & 0.34 & 0.32 & 0.35 & 0.34 \\
\hline & Rasulpur Marhi & 7.98 & 7.99 & 7.98 & 0.34 & 0.24 & 0.29 & 0.39 & 0.41 & 0.40 \\
\hline \multirow[t]{4}{*}{ Kharkhoda } & Mundali & 7.80 & 7.81 & 7.80 & 0.38 & 0.28 & 0.33 & 0.42 & 0.37 & 0.40 \\
\hline & Salempur & 7.46 & 7.48 & 7.47 & 0.39 & 0.30 & 0.35 & 0.48 & 0.44 & 0.46 \\
\hline & Phaphunda & 7.48 & 7.49 & 7.48 & 0.46 & 0.36 & 0.41 & 0.56 & 0.39 & 0.48 \\
\hline & Khandawali & 7.98 & 8.01 & 7.99 & 0.40 & 0.30 & 0.35 & 0.49 & 0.48 & 0.49 \\
\hline \multirow[t]{4}{*}{ Hashtinapur } & Palra & 7.98 & 7.98 & 7.98 & 0.31 & 0.21 & 0.26 & 0.39 & 0.36 & 0.38 \\
\hline & Tarapur & 7.45 & 7.46 & 7.45 & 0.31 & 0.22 & 0.27 & 0.38 & 0.43 & 0.41 \\
\hline & Pali & 7.80 & 7.81 & 7.80 & 0.41 & 0.31 & 0.36 & 0.29 & 0.37 & 0.33 \\
\hline & Ikwara, & 7.79 & 7.81 & 7.80 & 0.41 & 0.30 & 0.36 & 0.34 & 0.29 & 0.32 \\
\hline \multirow[t]{4}{*}{ Jani } & Afjal Pur Pawti & 7.79 & 7.79 & 7.79 & 0.38 & 0.28 & 0.33 & 0.32 & 0.38 & 0.35 \\
\hline & Pathanpura & 7.82 & 7.83 & 7.82 & 0.34 & 0.26 & 0.30 & 0.31 & 0.33 & 0.32 \\
\hline & Khanpur & 7.79 & 7.80 & 7.79 & 0.48 & 0.37 & 0.43 & 0.30 & 0.32 & 0.31 \\
\hline & Timkeya & 7.67 & 7.68 & 7.67 & 0.32 & 0.23 & 0.28 & 0.28 & 0.38 & 0.33 \\
\hline \multirow[t]{4}{*}{ Rajpura } & Shikhera & 8.05 & 8.08 & 8.06 & 0.31 & 0.20 & 0.26 & 0.39 & 0.32 & 0.36 \\
\hline & Mohiuddinpur & 7.96 & 7.96 & 7.96 & 0.41 & 0.31 & 0.36 & 0.36 & 0.38 & 0.37 \\
\hline & Uldeypur & 7.68 & 7.68 & 7.68 & 0.34 & 0.26 & 0.30 & 0.31 & 0.29 & 0.30 \\
\hline & Mamepur & 7.96 & 7.96 & 7.96 & 0.41 & 0.31 & 0.36 & 0.36 & 0.34 & 0.35 \\
\hline \multirow[t]{4}{*}{ Meerut } & Hajipur & 7.95 & 7.95 & 7.95 & 0.39 & 0.29 & 0.34 & 0.36 & 0.31 & 0.34 \\
\hline & Gagol & 8.03 & 8.04 & 8.03 & 0.46 & 0.37 & 0.42 & 0.36 & 0.28 & 0.32 \\
\hline & Azizpur & 7.95 & 7.95 & 7.95 & 0.32 & 0.22 & 0.27 & 0.29 & 0.41 & 0.35 \\
\hline & Shakarpur & 7.68 & 7.69 & 7.68 & 0.43 & 0.32 & 0.38 & 0.32 & 0.27 & 0.30 \\
\hline \multirow[t]{4}{*}{ Parikshitgarh } & Ahmadnagar Badhla & 7.81 & 7.82 & 7.81 & 0.40 & 0.30 & 0.35 & 0.44 & 0.41 & 0.43 \\
\hline & Alamgirpur Badhla & 7.21 & 7.25 & 7.23 & 0.42 & 0.32 & 0.37 & 0.33 & 0.40 & 0.37 \\
\hline & Chitwana Sherpur & 7.51 & 7.52 & 7.51 & 0.34 & 0.25 & 0.30 & 0.39 & 0.38 & 0.39 \\
\hline & Poothi & 7.82 & 7.82 & 7.82 & 0.43 & 0.34 & 0.39 & 0.34 & 0.33 & 0.34 \\
\hline \multirow[t]{4}{*}{ Machara } & Hasanpur & 7.40 & 7.42 & 7.41 & 0.48 & 0.39 & 0.44 & 0.44 & 0.41 & 0.43 \\
\hline & Bharolli & 7.76 & 7.77 & 7.76 & 0.42 & 0.33 & 0.38 & 0.36 & 0.37 & 0.37 \\
\hline & Amarpur & 7.97 & 7.98 & 7.97 & 0.31 & 0.21 & 0.26 & 0.34 & 0.33 & 0.34 \\
\hline & Alipur Alampur & 7.65 & 7.65 & 7.65 & 0.44 & 0.34 & 0.39 & 0.33 & 0.37 & 0.35 \\
\hline Mavana & Amroli & 7.66 & 7.68 & 7.67 & 0.43 & 0.33 & 0.38 & 0.36 & 0.33 & 0.35 \\
\hline & Bhainsa & 7.90 & 7.92 & 7.91 & 0.41 & 0.32 & 0.37 & 0.31 & 0.35 & 0.33 \\
\hline & Bhagawanpur & 7.94 & 7.96 & 7.95 & 0.40 & 0.30 & 0.35 & 0.29 & 0.40 & 0.35 \\
\hline & Rahawati & 7.83 & 7.85 & 7.84 & 0.31 & 0.20 & 0.26 & 0.30 & 0.31 & 0.31 \\
\hline & Mean & 7.76 & 7.77 & - & 0.38 & 0.28 & - & 0.35 & 0.36 & - \\
\hline
\end{tabular}


Depth Wise Variability of Available N, P and K at Different Locations under Rice - Pea- Sugarcane Cropping System in Meerut...

Table 3: Depth wise availability of $\mathrm{N}, \mathrm{P}$ and $\mathrm{K}(\mathrm{kg} / \mathrm{ha})$ of different location soil under rice -pea - sugarcane cropping system.

\begin{tabular}{|c|c|c|c|c|c|c|c|c|c|c|}
\hline \multicolumn{2}{|c|}{ Location } & \multicolumn{9}{|c|}{ Cropping system (Rice - pea - sugarcane) and Depth (cm) } \\
\hline \multirow{2}{*}{ Blocks name } & \multirow{2}{*}{ Village name } & \multicolumn{3}{|c|}{ Available $\mathrm{N}(\mathrm{kg} / \mathrm{ha})$} & \multicolumn{3}{|c|}{$P(\mathrm{~kg} / \mathrm{ha})$} & \multicolumn{3}{|c|}{$\mathrm{K}(\mathrm{kg} / \mathrm{ha})$} \\
\hline & & $0-15$ & $15-30$ & Mean & $0-15$ & $15-30$ & Mean & $0-15$ & $15-30$ & Mean \\
\hline \multirow[t]{4}{*}{ Daurala } & Lawar Khas & 174.89 & 107.89 & 141.39 & 17.89 & 13.71 & 15.80 & 170.66 & 106.23 & 138.45 \\
\hline & Lawar Khas & 169.98 & 109.89 & 139.94 & 16.82 & 12.21 & 14.52 & 182.63 & 110.11 & 146.37 \\
\hline & Akhtyarpur & 185.89 & 109.97 & 147.93 & 18.23 & 13.73 & 15.98 & 184.94 & 113.47 & 149.21 \\
\hline & Chindauri & 246.44 & 166.54 & 206.49 & 31.51 & 21.12 & 26.32 & 235.20 & 165.36 & 200.28 \\
\hline \multirow[t]{4}{*}{ Shardhana } & Chabariya & 202.42 & 128.63 & 165.53 & 21.22 & 15.51 & 18.37 & 201.80 & 112.21 & 157.01 \\
\hline & Begumabad & 208.00 & 134.21 & 171.11 & 24.32 & 16.00 & 20.16 & 204.32 & 116.86 & 160.59 \\
\hline & Milak & 201.23 & 125.54 & 163.39 & 24.45 & 15.35 & 19.90 & 193.20 & 110.86 & 152.03 \\
\hline & Teherkiya & 234.10 & 157.89 & 196.00 & 29.67 & 18.99 & 24.33 & 227.50 & 130.60 & 179.05 \\
\hline \multirow[t]{4}{*}{ Sururpur } & Saroorpur Khurd & 211.78 & 138.88 & 175.33 & 28.16 & 16.82 & 22.49 & 209.86 & 140.60 & 175.23 \\
\hline & Kheri Kalan, & 203.68 & 128.88 & 166.28 & 25.83 & 15.57 & 20.70 & 203.32 & 125.96 & 164.64 \\
\hline & Harar & 213.25 & 141.87 & 177.56 & 24.44 & 14.86 & 19.65 & 210.76 & 136.02 & 173.39 \\
\hline & Sultannagar & 255.90 & 180.13 & 218.02 & 33.66 & 21.98 & 27.82 & 248.56 & 185.12 & 216.84 \\
\hline \multirow[t]{4}{*}{ Rohta } & Rasulpur & 227.31 & 155.27 & 191.29 & 27.63 & 18.36 & 23.00 & 221.50 & 125.87 & 173.69 \\
\hline & Rasna & 252.13 & 171.13 & 211.63 & 27.42 & 21.98 & 24.70 & 238.72 & 152.00 & 195.36 \\
\hline & Bhadaura & 216.78 & 144.10 & 180.44 & 22.89 & 17.44 & 20.17 & 214.08 & 122.22 & 168.15 \\
\hline & Rasulpur Marhi & 207.86 & 133.54 & 170.70 & 26.94 & 15.88 & 21.41 & 203.52 & 116.86 & 160.19 \\
\hline \multirow[t]{4}{*}{ Kharkhoda } & Mundali & 216.00 & 143.89 & 179.95 & 23.76 & 17.38 & 20.57 & 213.43 & 121.53 & 167.48 \\
\hline & Salempur & 217.63 & 145.06 & 181.35 & 28.47 & 17.51 & 22.99 & 214.54 & 123.31 & 168.93 \\
\hline & Phaphunda & 246.74 & 167.06 & 206.90 & 28.10 & 21.34 & 24.72 & 235.20 & 162.45 & 198.83 \\
\hline & Khandawali & 217.68 & 145.73 & 181.71 & 21.98 & 17.76 & 19.87 & 218.40 & 123.65 & 171.03 \\
\hline \multirow[t]{4}{*}{ Hashtinapur } & Palra & 189.96 & 119.89 & 154.93 & 23.16 & 14.90 & 19.03 & 189.87 & 105.75 & 147.81 \\
\hline & Tarapur & 201.12 & 125.32 & 163.22 & 19.74 & 15.12 & 17.43 & 192.87 & 110.60 & 151.74 \\
\hline & Pali & 220.34 & 150.13 & 185.24 & 22.76 & 18.10 & 20.43 & 218.68 & 125.31 & 172.00 \\
\hline & Ikwara, & 220.21 & 149.59 & 184.90 & 28.48 & 18.05 & 23.27 & 218.68 & 150.76 & 184.72 \\
\hline \multirow[t]{4}{*}{ Jani } & Afjal Pur Pawti & 215.20 & 143.23 & 179.22 & 28.16 & 17.35 & 22.76 & 212.54 & 120.77 & 166.66 \\
\hline & Pathanpura & 209.95 & 136.76 & 173.36 & 27.38 & 16.36 & 21.87 & 205.45 & 139.51 & 172.48 \\
\hline & Khanpur & 247.56 & 170.21 & 208.89 & 32.12 & 21.54 & 26.83 & 235.20 & 136.83 & 186.02 \\
\hline & Timkeya & 207.65 & 132.42 & 170.04 & 26.87 & 15.88 & 21.38 & 203.52 & 136.66 & 170.09 \\
\hline \multirow[t]{4}{*}{ Rajpura } & Shikhera & 188.16 & 114.9 & 151.53 & 21.10 & 13.94 & 17.52 & 184.94 & 116.10 & 150.52 \\
\hline & Mohiuddinpur & 223.32 & 152.43 & 187.88 & 28.50 & 18.22 & 23.36 & 220.32 & 139.84 & 180.08 \\
\hline & Uldeypur & 210.12 & 137.65 & 173.89 & 27.72 & 16.74 & 22.23 & 206.44 & 134.61 & 170.53 \\
\hline & Mamepur & 224.78 & 153.32 & 189.05 & 26.52 & 18.35 & 22.44 & 220.32 & 141.48 & 180.90 \\
\hline \multirow[t]{4}{*}{ Meerut } & Hajipur & 216.82 & 144.76 & 180.79 & 28.47 & 17.49 & 22.98 & 214.08 & 123.09 & 168.59 \\
\hline & Gagol & 247.32 & 167.77 & 207.55 & 22.17 & 21.47 & 21.82 & 235.20 & 136.80 & 186.00 \\
\hline & Azizpur & 204.60 & 132.32 & 168.46 & 22.43 & 15.57 & 19.00 & 203.34 & 128.48 & 165.91 \\
\hline & Shakarpur & 240.40 & 162.18 & 201.29 & 31.13 & 19.00 & 25.07 & 231.56 & 148.66 & 190.11 \\
\hline \multirow[t]{4}{*}{ Parikshitgarh } & Ahmadnagar Badhla & 218.23 & 146.98 & 182.61 & 26.74 & 17.89 & 22.32 & 218.60 & 124.77 & 171.69 \\
\hline & Alamgirpur Badhla & 233.43 & 157.84 & 195.64 & 22.78 & 18.91 & 20.85 & 224.54 & 147.16 & 185.85 \\
\hline & Chitwana Sherpur & 208.90 & 135.43 & 172.17 & 27.38 & 16.15 & 21.77 & 204.35 & 136.48 & 170.42 \\
\hline & Poothi & 240.40 & 163.78 & 202.09 & 31.13 & 19.65 & 25.39 & 231.56 & 151.70 & 191.63 \\
\hline \multirow[t]{4}{*}{ Machara } & Hasanpur & 254.23 & 178.10 & 216.17 & 32.21 & 21.98 & 27.10 & 246.40 & 139.11 & 192.76 \\
\hline & Bharolli & 238.32 & 162.43 & 200.38 & 29.84 & 19.04 & 24.44 & 228.36 & 157.3 & 192.83 \\
\hline & Amarpur & 194.87 & 121.45 & 158.16 & 23.50 & 15.07 & 19.29 & 189.98 & 105.86 & 147.92 \\
\hline & Alipur Alampur & 243.44 & 165.78 & 204.61 & 28.94 & 20.43 & 24.69 & 234.56 & 156.72 & 195.64 \\
\hline Mavana & Amroli & 238.45 & 163.24 & 200.85 & 27.76 & 19.05 & 23.41 & 228.36 & 132.75 & 180.56 \\
\hline & Bhainsa & 232.20 & 156.87 & 194.54 & 26.71 & 18.89 & 22.80 & 224.00 & 138.70 & 181.35 \\
\hline & Bhagawanpur & 218.54 & 148.72 & 183.63 & 18.98 & 18.01 & 18.50 & 218.60 & 141.76 & 180.18 \\
\hline & Rahawati & 189.87 & 115.79 & 152.83 & 22.91 & 14.57 & 18.74 & 188.95 & 103.20 & 146.08 \\
\hline & Mean & 218.50 & 144.70 & - & 25.81 & 17.53 & - & 213.82 & 131.92 & - \\
\hline
\end{tabular}


which might be due to the accumulation of plant residues, debris at the surface which contributed through mineralization. These observations are following the findings of Prasuna Rani et al. (1992). Correlation studies show that a significant positive correlation was found between organic carbon and available nitrogen. This might be because most of the soil nitrogen is found in organic forms. Similar results were also reported by Kanthalia and Bhatt (1991); Paliwal (1996).

The available phosphorus $\left(\mathrm{P}_{2} \mathrm{O}_{5}\right)$ in the surface $(0-15$ $\mathrm{cm})$ and subsurface $(15-30 \mathrm{~cm})$ varied from 16.82 to 33.66 and 12.21 to $21.98 \mathrm{~kg} \mathrm{ha}^{-1} \mathrm{P}_{2} \mathrm{O}_{5}$, respectively (Table 3 ). The maximum available phosphorus $33.66 \mathrm{~kg} \mathrm{ha}^{-1} \mathrm{P}_{2} \mathrm{O}_{5}$ was found in Sultannagar village of Sururpur block and minimum $16.82 \mathrm{P}_{2} \mathrm{O}_{5} \mathrm{~kg} \mathrm{ha}^{-1}$ in Lawar Khas location at the surface (0$15 \mathrm{~cm})$, whereas, at subsurface $(15-30 \mathrm{~cm})$ maximum 21.98 $\mathrm{P}_{2} \mathrm{O}_{5} \mathrm{~kg} \mathrm{ha}^{-1}$ was found in Sultannagar location and minimum 12.21 $\mathrm{P}_{2} \mathrm{O}_{5} \mathrm{~kg} \mathrm{ha}^{-1}$ in Lawar Khas location. The range is quite large which might be due to variation of soil properties viz., $\mathrm{pH}$, calcareousness, organic matter content, texture and various soil management and agronomic practices. Based on the limit suggested by Muhr et al., (1968) $42 \%$ of samples were rated low $\left(<25 \mathrm{P}_{2} \mathrm{O}_{5} \mathrm{~kg} \mathrm{ha}^{-1}\right)$ and $58 \%$ of samples were medium (16-35 $\mathrm{P}_{2} \mathrm{O}_{5} \mathrm{~kg} \mathrm{ha}^{-1}$ ). However, the highest available phosphorus was observed in the surface horizons and decreased regularly with depth. Higher phosphorus in the surface horizons might be due to the confinement of crop cultivation to this layer and supplement of the depleted phosphorus through external sources i.e. fertilizers (Rajeswar et al., 2009). Similar results were reported by Thangaswamy et al. (2005).

Available potassium $\left(\mathrm{K}_{2} \mathrm{O}\right)$ surface $(0-15 \mathrm{~cm})$ and subsurface $(15-30 \mathrm{~cm})$ ranged between 170.66 to 248.56 and 103.20 to $185.12 \mathrm{~kg} \mathrm{ha}^{-1} \mathrm{~K}_{2} \mathrm{O}$ (Table 3). The maximum available potassium $248.56 \mathrm{~kg} \mathrm{ha}^{-1} \mathrm{~K}_{2} \mathrm{O}$ was found at Sultannagar and a minimum of $170.66 \mathrm{~kg} \mathrm{ha}^{-1} \mathrm{~K}_{2} \mathrm{O}$ in Lawar Khas location at the surface $(0-15 \mathrm{~cm})$. Similarly, maximum available potassium $185.12 \mathrm{~K}_{2} \mathrm{O} \mathrm{kg} \mathrm{ha-1}$ was found in Sultannagar and minimum $103.20 \mathrm{~K}_{2} \mathrm{O} \mathrm{kg} \mathrm{ha}{ }^{-1}$ Rahawati locations at subsurface $(15-30 \mathrm{~cm})$. Due to unawareness about potassium application and more removal from soils by crops available $\mathrm{K}$ is lower. In grasslands, wastes land and forest soils, probably loss $\mathrm{K}$ removal by plants, maintained a comparatively higher $\mathrm{K}$ in soils (Choudhary Ram 2005). According to Muhr et al. (1963), no sample was found in the low range $\left(<125 \mathrm{~K}_{2} \mathrm{O} \mathrm{kg} \mathrm{ha-1}\right)$ however were the medium range $\left(125-300 \mathrm{~kg} \mathrm{ha}^{-1} \mathrm{~K}_{2} \mathrm{O}\right.$ the surface $(0-15 \mathrm{~cm})$ and Subsurface (15-30 cm). A significant positive correlation was observed between organic carbon and available potassium content. This might be due to the creation of a favorable soil environment with the presence of high organic matter. Similar results were also reported by Paliwal (1996); Chouhan (2001).

\section{Relationship of $\mathrm{pH}$ and $\mathrm{OC}$ with soil properties}

Relationships of $\mathrm{pH}$ and Organic carbon with some soil properties like Available N, P and K were established by the determination of correlation coefficient values (Table 1 ).
OC significantly and positively correlated with the above discussed soil properties $(r=0.952,0.658,0.960$ and subsurface, $r=0.983,0.973$ and 0.795 respectively).

The analysis indicates that $\mathrm{pH}$ was negatively correlated with Available N, P and $K$ in surface $(0-15 \mathrm{~cm})$ and subsurface $(15-30 \mathrm{~cm})$.

\section{CONCLUSION}

In-depth wise availability was affected by Rice - PeaSugarcane cropping system and the properties of soil indicate that the soils of the study area were neutral to strongly alkaline in reaction and non-saline in nature. Nutrient status in surface and subsurface depth of soil indicates that soils are low in available $\mathrm{N}$ and medium in available $P$ and available $K$ in surface and subsurface soil layer of the profiles. Relationships of $\mathrm{pH}$ and Organic carbon with some soil properties like Available N, P and $\mathrm{K}$ were established by determination of correlation coefficient. The analysis indicates that $\mathrm{pH}$ was negatively correlated with Available N, P, K similar results were observed by Tisdale et al., 1997, Similar results were also noticed by Singh 1988 in the soils of Udaipur. OC significantly and positively correlated with the above-discussed soil properties. So the cropping system is useful for soil health sustainability.

\section{REFERENCES}

Ali, M., Gupta, S. (2012). Carrying capacity of Indian agriculture: Pulse crops. Current Sci. 102: 874-881.

Chaudhary, Ram. (2005). Effect of cutting management and seed yield of the grass-legume mixture under dry temperate grassland of Lahaul and Spiti (HP), Ph.D. Thesis, CSKHPKV, Palampur.

Chauhan, B.S., Mahajan, G., Sardana, V., Timsina, J., Jat, M.L. (2012). Productivity and sustainability of the rice-wheat cropping system in the Indo-Gangetic Plains of the Indian subcontinent: Problems, opportunities and strategies. Adv Agron. 117: 315-369.

Chouhan, J.S., (2001). Fertility Status of Soils of Bilara Panchayat Samiti of Jodhpur district (Rajsthan). M.Sc. (Ag.) Thesis, MPUAT, Udaipur.

Jackson, M.L. (1973). Soil Chemical Analysis Prentice Hall of India Pvt. Ltd., New Delhi.

Kathaliya, P.C., Bhatt, P.C. (1991). Relation between organic carbo and available nutrients in some soils of the sub-humid zone. Journal of Indian Society of Soil Science. 39: 781-782.

Ladha, J.K., Pathak, H., Tirol, Padre, A., Dawe, D., Gupta, R.K. (2003). Productivity trends in intensive rice-wheat cropping systems in Asia. Improving the productivity and sustainability of rice-wheat systems: Issues and impacts. Madison, Wisconsin: USA: Agronomy Series ASA-CSSA-SSSA Publishers. p. 45-76.

Muhr, G.R., Datta, N.P., Shankara, S.N., Dever, F., Lecy, V.K., Donahue, R.R. (1963). Soil testing in India.USDA Mission to India.

Olsen, S.R., Cole, C.V., Watanabe, F.S. and Dean, L.A. (1954). Estimation of available phosphorus in soils by extraction with sodium bicarbonate. United States Department of Agriculture Circular. 9: 19-39. 
Paliwal, M.L. (1996). Studied on major and micronutrient status of soils of panchayat Samiti Bhinder (Dist. Udaypur). M.Sc. (Ag.) Thesis, Rajasthan Agricultural University, Bikaner.

Parsuna Rani, P.P., Pillai, R.N., Bhanu Prasad, V., Subbaiah, G.V. (1992). Nutrient status of Some red and associated soils of Nellore District in Somasila project in Andhra Pradesh. The Andhra Agricultural Journal. 49: 228-236.

Rajeshwar, M., Rao, Sujini, Ch., Balaguravaiah, D., Arif, Khan, M.A. (2009). Distribution of available macro and micronutrients in soils of Garikapadu of Krishna district of Andhra Pradesh. Journal of the Indian Society of Soil Science. 57: 210-213.

Singh, L. (1988). Studied on major and micronutrient status of soils of panchayat Samiti Bhinder (Dist. Udaipur). M.Sc. (Ag.) Thesis, Rajasthan Agricultural University, Bikaner.

Singh, R.K., Singh, S.K. and Tarafdar, J.C. (2008). Influence of cropping sequence and nutrient management on soils organic carbon and nutrient status of Typical Rhodustalalls. Journal of the Indian Society of Soil Science. 56: 174-181.
Subbiah, BV., Asija, G.L. (1956). A rapid procedure for the determination of available nitrogen in the oil. Current Science. 25: 259-260.

Thangaswamy, A., Naidu, M.V.S., Ramavatharam, M., Raghavereddy, C. (2005). Characterization, classification and evaluation of soil resources in Sivagiri Micro-the watershed of Chittor District in Andhara Pradesh for sustainable land use planning. Journal of the Indian Society of Soil Science. 53: 11-21.

Thangaswamy, A., Naidu, M.V.S., Ramavatharam, M., Raghavereddy, C. (2005). Characterization, classification and evaluation of soil resources in Sivagiri Micro- the watershed of Chittor District in Andhara Pradesh for sustainable land use planning. Journal of the Indian Society of Soil Science. 53: 11-21.

Tisdale, S.L. Nelson, W.L. Beaton, J.D., Havlin, J.L. (1997). Soil Fertility and Fertilizer, $5^{\text {th }}$ Edition, Macmillian Publishing Co., New Delhi. 144,180,198,201.

Walkley, A.J., Black, I.A. (1934). Estimation of soil organic carbon by the chromic acid titration method. Soil Science. 37 : 29-38. 